\title{
Good local tumor control but lethal hemorrhage after apatinib treatment for intractable squamous carcinoma of the floor of the mouth: a case report
}

This article was published in the following Dove Press journal: OncoTargets and Therapy

\author{
Xiaopeng Zhu',* \\ Min Liul,* \\ Jincai Lv' \\ Liang Guo \\ Cheng Li' \\ Lihua Dong' \\ Bailong Liu' \\ 'Department of Radiation Oncology, \\ The First Hospital, Jilin University, \\ Changchun I3002I, China; \\ ${ }^{2}$ Department of Pathology, The First \\ Hospital, Jilin University, Changchun \\ |3002 I, China \\ *These authors contributed equally \\ to this work
}

\begin{abstract}
The treatment of repeatedly recurrent carcinoma of the floor of the mouth (FOM) is challenging. There is no standard strategy for such patients with poor physical condition after multiple lines of treatment. Angiogenesis is a key in tumor initiation, growth, and dissemination. Apatinib, a potent tyrosine kinase inhibitor targeting vascular endothelial growth factor receptor 2 (VEGFR2), has been approved for the treatment of late-stage gastric or gastroesophageal junction adenocarcinoma that is resistant to at least two lines of chemotherapy. Its application in intractable FOM squamous carcinoma has never been described before. Herein, we present the case of a heavily treated patient with FOM squamous carcinoma undergoing a third local relapse in the right region of the neck and anterior cervical region. Oral apatinib was administered daily at a dose of $250 \mathrm{mg}$. There was clear and rapid efficacy that led to complete remission. However, giant, deep ulcers formed due to tumor necrosis. The patient eventually died of massive bleeding resulting from the major cervical vascular rupture caused by tumor necrosis and erosion. This case is novel and instructional, highlighting that apatinib might be effective, with manageable toxicity, for certain patients with refractory head and neck squamous cell carcinoma (HNSCC). The advantages and disadvantages of apatinib should be carefully evaluated, and close surveillance and quick intervention as required are critical to reduce fatal cancer-associated complications. The role of apatinib in recurrent or metastatic HNSCC needs to be clarified by multicenter trials in the near future.
\end{abstract}

Keywords: apatinib, head and neck squamous cell carcinoma, recurrent, lethal bleeding, VEGFR2, TKI

\section{Introduction}

Carcinoma originating from the floor of the mouth (FOM; 27.2\%) is the second most common oral cancer, second only to tongue cancer $(35.1 \%) .{ }^{1-3}$ FOM squamous carcinoma poses special clinical concerns owing to the limited surgical access because of the narrow anatomic space, esthetic and functional requirements, and high tendency for cervical lymph node metastasis. ${ }^{1}$ With the advancements of comprehensive antitumor treatment and the prolongation of survival, relapse and/or metastasis is common, especially in the heavily pretreated population who have developed resistance to the conventional chemotherapeutics and radiotherapy. New treatment strategies with large antitumor effects and good tolerance are urgently required. The role of antiangiogenic drugs in recurrent or metastatic $(\mathrm{R} / \mathrm{M})$ head and neck squamous cell carcinoma (HNSCC) needs to be further identified and might give rise to new insights in the near future.
Correspondence: Lihua Dong; Bailong Liu Department of Radiation Oncology, The First Hospital, Jilin University, 7I Xinmin Street, Changchun, I3002I, China

Tel +86 I58 430732 I6;

+8615804302752

Email drlhdong@163.com; liubl@jlu.edu.cn 


\section{Case report}

A 49-year-old Chinese male was admitted to a tertiary hospital in May 2013 due to a 2-month history of a progressively developing mass in the right region of the FOM. The biopsy conducted in the outpatient clinic demonstrated moderately differentiated squamous carcinoma. Local resection with a $0.5-\mathrm{cm}$ margin was conducted. The patient was staged as pT1Nx. However, in view of the inadequate borders and the lack of neck lymph node dissection, concurrent radiotherapy with weekly cisplatin administration was performed in our hospital postoperatively to improve local control after communicating with the first surgeon.

In November 2015, the patient experienced a recurrence in a region in the right of the neck. Surgery involving dissection of the right II-V lymphatic drainage areas was performed. The lymph nodes were found to be negative for metastases (0/25), but cancerous nodes without lymph node structure and with a maximum diameter of $2.8 \mathrm{~cm}$ were found at level III in the right region of the neck, invading the striated muscle tissue and nerves. In August 2016, the mass in the right region of the neck recurred for the second time and progressed aggressively. A subsequent computed tomography (CT) scan indicated multiple enlarged lymph nodes located in the right region of the neck at levels III-VI, without a clear boundary with the right common carotid artery (Figure $1 \mathrm{~A}$ and B). After a cycle of induction chemotherapy (docetaxel+nedaplatin), reirradiation with $70 \mathrm{~Gy} / 35 \mathrm{f}$ to the metastatic lymph nodes and $50 \mathrm{~Gy} / 25 \mathrm{f}$ to the high-risk neck region concurrently with three weekly cycles of nedaplatin contributed to the complete remission (CR) response (Figure 1C).

In January 2018, the patient experienced a third regional relapse in the right region of the neck again (Figure 2A). The tumor grew rapidly, extending to the anterior cervical region, and was cauliflower-like or nodular with surface bleeding and exudation (Figure 2B). At this point, the patient refused chemotherapy, and he could not afford immune checkpoint inhibitors. In consideration of cost-effectiveness, tolerance, and availability, apatinib, a small-molecule tyrosine kinase inhibitor (TKI) targeting vascular endothelial growth factor receptor 2 (VEGFR2), was initiated at a daily dose of $250 \mathrm{mg}$. After only 7 days of use, the tumor shrank dramatically (Figure 2C). A CR response was attained after taking apatinib for 20 days. However, deep local ulcers formed due to the rapid tumor shrinkage (Figure 2D), and apatinib was suspended. Two weeks later, the tumor had grown aggressively (Figure 2E). The patient restarted $250 \mathrm{mg}$ apatinib daily for about 3 weeks and experienced a CR response again. However, giant infected ulcers emerged (Figure 2F).
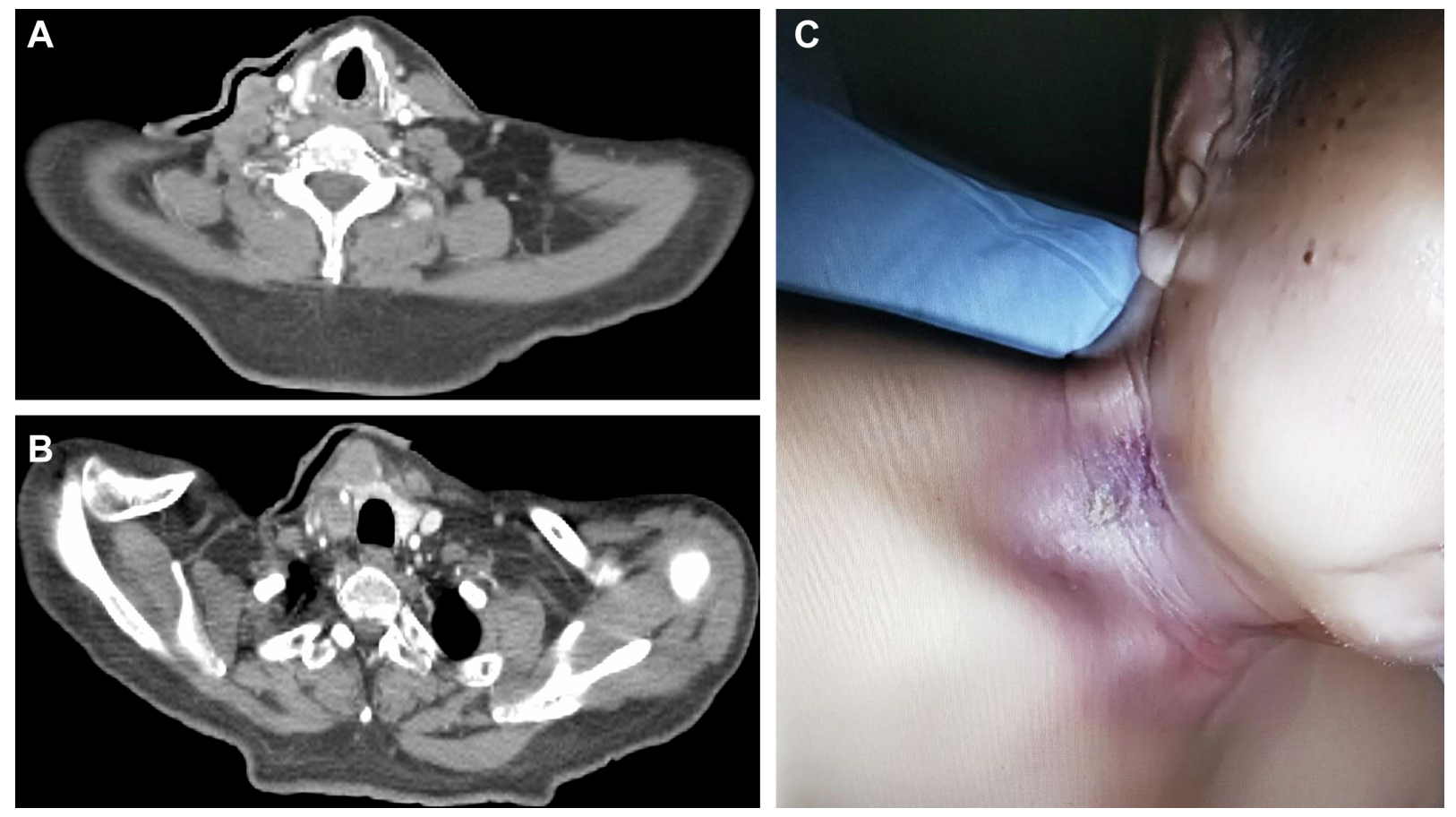

Figure I The neck CT scan showed multiple metastatic cervical lymph nodes located in the right III, IV, V, and VI regions, with no clear boundary with the right common carotid artery at the second local regional relapse (A and B). After induction chemotherapy and definitive reirradiation with synchronized weekly chemotherapy, the patient experienced complete remission (C).

Abbreviation: CT, computed tomography. 

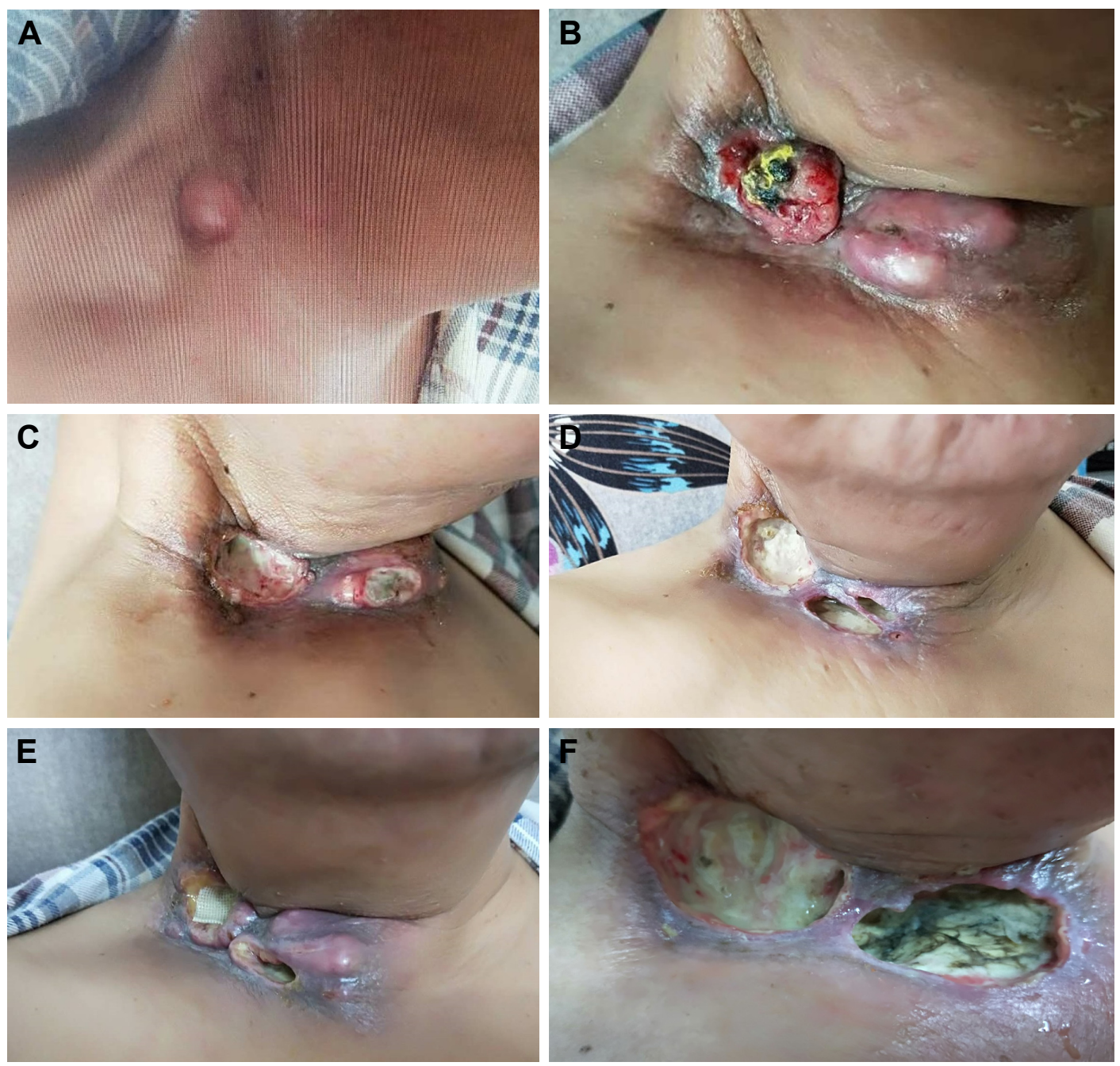

Figure 2 The process of tumor change and ulcer formation during the application of apatinib, after the third local regional recurrence.

Notes: In January 2018, the patient experienced a third recurrence in the right region of the neck (A). The tumor progressed aggressively during a 2-month period before the initiation of apatinib (B). The tumor shrank significantly after 7 days of $250 \mathrm{mg}$ apatinib daily (C). The 20-day use of apatinib contributed to complete remission, but deep ulcers formed owing to tumor regression (D). After a 2-week suspension of apatinib, the tumor regrew (E). The tumor disappeared after a 3-week reapplication of apatinib, but large ulcers with local infection occurred (F).

The wound was rinsed with a solution containing gentamicin and metronidazole, but there was little improvement. The CT scans revealed huge tissue defects, with invasion and exposure of the right carotid vein and artery (Figure 3). The other side effects related to apatinib were mild except for grade 2 hypertension (which was easily controlled by blood pressurelowering drugs) and fatigue. Unfortunately, the patient died due to major bleeding caused by rupture of the right neck vessels on May 22, 2018.

This study was approved by the ethics committee of the First Hospital of Jilin University, and written informed consent was obtained from the patient for publication of this case report and accompanying images.

\section{Discussion}

Repeated recurrent FOM carcinoma is a thorny clinical problem. Patients generally have a poor performance status after undergoing multiple lines of chemotherapy and radiotherapy, and they become incapable of undergoing further intense intervention. Therapeutic options in such circumstances are limited to treatments with mild efficacy. Our patient experienced regional failure three times in the course of undergoing surgery twice and radiation combined with chemotherapy twice.

Targeted drugs have the advantages of good tolerance, convenient use, and efficacy. Alternative evidence-based targeted drugs for our patient might have included cetuximab, afatinib, and nivolumab. EGFR is overexpressed in $90 \%$ of 


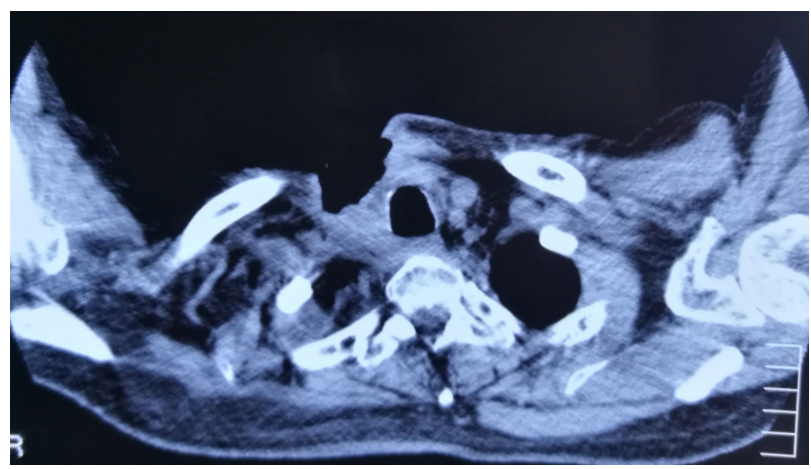

Figure 3 The CT scan conducted on May 17, 2018, revealed obvious tissue defects in the right lower neck and anterior cervical region, with no clear boundaries with the right carotid artery and vein.

Abbreviation: CT, computed tomography.

cases of HNSCC. ${ }^{4}$ The anti-EGFR antibody cetuximab does not have a major role as a monotherapy, and it is generally combined with other modalities such as radiotherapy or chemotherapy. ${ }^{5}$ Caution should be taken during the clinical application of cetuximab due to its serious side effects such as interstitial lung disease and infusion reaction (which is unique to antibody therapy). ${ }^{5}$ Afatinib, an irreversible inhibitor of ErbB family proteins (which are receptor tyrosine kinases, structurally related to EGFR), has comparable antitumor efficacy to cetuximab but causes more frequent severe adverse events in patients with R/M HNSCC refractory to platinum-containing regimens. ${ }^{6}$ As a second-line option for these patients, afatinib achieved a median progression-free survival (PFS) of 2.6 months. $^{7}$ Nivolumab, a monoclonal antibody targeting programmed cell death protein 1 (PD-1), prolonged the median overall survival (OS) compared to that by standard monotherapy involving docetaxel, methotrexate, or cetuximab (7.5 vs 5.1 months) in patients with $\mathrm{R} / \mathrm{M}$ HNSCC not amenable to curative treatment. ${ }^{8}$

Angiogenesis is essential and remains active during tumor development. ${ }^{9}$ Apatinib, a novel small-molecule TKI targeting an intracellular segment of VEGFR2, has been approved for recurrent gastric or gastroesophageal junction adenocarcinoma after at least two lines of chemotherapy. In addition, clinical trials have verified the safety and efficacy of apatinib in other R/M or refractory cancers, including breast cancer, ${ }^{10}$ lung cancer, ${ }^{11}$ colorectal cancer, ${ }^{12}$ non-Hodgkin lymphoma, ${ }^{13}$ sarcoma, ${ }^{14,15}$ and epithelial ovarian cancer. ${ }^{16}$ However, its application in R/M HNSCC has seldom been described. In the report by $\mathrm{Wu}$ et al, apatinib alone achieved an objective response rate (ORR) of $25 \%$ and a disease control rate (DCR) of $50 \%$ in eight patients with advanced HNSCC who did not respond adequately to standard chemotherapy or chemoradiotherapy. The side effects were manageable; $25 \%$ were grade $3 / 4$ treatment-associated adverse events, mainly hypertension. ${ }^{17}$ Furthermore, in five patients with $\mathrm{R} / \mathrm{M}$ oral squamous cell carcinoma refractory to chemotherapy, apatinib alone attained an ORR of $20 \%$ and DCR of $60 \%{ }^{18}$ Compared with other target drugs, apatinib has a high cost-effectiveness and availability. To the best of our knowledge, this is the first case report of apatinib use in a heavily pretreated patient with refractory FOM squamous carcinoma. Undoubtedly, apatinib exerted a rapid and overwhelming tumor control effect. However, the ulcers that developed due to tumor necrosis were giant and deep, and local infection of these ulcers occurred. We are familiar with the common side effects of apatinib such as hypertension, proteinuria, hand-foot syndrome, and fatigue, which are generally acceptable and manageable. However, life-threatening hemorrhage caused by tumor necrosis and erosion during the use of apatinib has seldom been documented. After extensive review of the literature, only one report was found. Wang et al described two patients with advanced esophageal cancer treated with apatinib who died of hemoptysis. The tumors responded remarkably to apatinib, but local cavities were formed. Rupture of the bronchial artery that had been eroded by the tumor resulted in the massive bleeding. ${ }^{19}$

The findings related to this case are noteworthy for multiple reasons. First, apatinib is highly valid for some R/M HNSCC patients who are resistant to standard chemotherapy regimens and radiotherapy. As tumors are sustained with nutrition and oxygen supplied by neovascularization, antiangiogenesis therapy might be a good option for such a difficult situation, especially for tumors that are rich in blood vessels and growing fast. However, as this is only a case report and only small sample clinical studies have been performed to support this view, ${ }^{17,18}$ in the near future, the role of apatinib in R/M HNSCC needs to be clarified by multicenter trials. In addition, biomarkers predicting the effect of apatinib should be further explored. Second, careful assessment of blood vessels eroded by the tumor is essential. CT angiography might be helpful. If the tumor has invaded the entire vessel wall layer, especially the key large blood vessels, the patient is at high risk of fatal vascular rupture and bleeding. According to the degree and extent of erosion, interventions such as vascular embolism can be considered to reduce the risk of massive hemorrhage. Third, as an antiangiogenic drug, apatinib affects the repair of surrounding normal tissues in addition to reducing the tumor blood supply. Apatinib should be temporarily suspended before and 30 days after operation. In addition, in our case, infection reduced the repair and healing of the normal tissue. Active anti-infection therapy 
and methods to promote the repair of normal tissues are of great significance.

\section{Conclusion}

Apatinib might be beneficial for some R/M HNSCC patients who are refractory to multiple treatments. However, special attention should be paid to the possibility of massive bleeding caused by tumor erosion and necrosis. Detailed assessment, close surveillance, and active management are important to minimize lethal cancer-related complications besides yielding ideal tumor control. The role of apatinib in $\mathrm{R} / \mathrm{M}$ HNSCC needs to be identified by large-scale prospective clinical trials in the future.

\section{Disclosure}

The authors report no conflicts of interest in this work.

\section{References}

1. Saggi S, Badran KW, Han AY, Kuan EC, St John MA. Clinicopathologic characteristics and survival outcomes in floor of mouth squamous cell carcinoma: a population-based study. Otolaryngol Head Neck Surg. 2018;159(1):51-58.

2. Funk GF, Karnell LH, Robinson RA, Zhen WK, Trask DK, Hoffman HT. Presentation, treatment, and outcome of oral cavity cancer: a national cancer data base report. Head Neck. 2002;24(2):165-180.

3. Li R, Fakhry C, Koch WM, Gourin CG. The Effect of tumor subsite on short-term outcomes and costs of care after oral cancer surgery. Laryngoscope. 2013;123(7):1652-1659.

4. Bancroft CC, Chen Z, Yeh J, et al. Effects of pharmacologic antagonists of epidermal growth factor receptor, PI3K and MEK signal kinases on NF-kappaB and AP-1 activation and IL-8 and VEGF expression in human head and neck squamous cell carcinoma lines. Int J Cancer. 2002; 99(4):538-548.

5. Nibu KI, Hayashi R, Asakage T, et al. Japanese clinical practice guideline for head and neck cancer. Auris Nasus Larynx. 2017;44(4):375-380.

6. Seiwert TY, Fayette J, Cupissol D, et al. A randomized, phase II study of afatinib versus cetuximab in metastatic or recurrent squamous cell carcinoma of the head and neck. Ann Oncol. 2014;25(9):1813-1820.
7. Machiels JP, Haddad RI, Fayette J, et al. Afatinib versus methotrexate as second-line treatment in patients with recurrent or metastatic squamous-cell carcinoma of the head and neck progressing on or after platinum-based therapy (LUX-Head \& Neck 1): an open-label, randomised phase 3 trial. Lancet Oncol. 2015;16(5):583-594.

8. Ferris RL, Blumenschein G, Fayette J, et al. Nivolumab for recurrent squamous-cell carcinoma of the head and neck. $N$ Engl J Med. 2016; 375(19):1856-1867.

9. Hanahan D, Weinberg RA. Hallmarks of cancer: the next generation. Cell. 2011;144(5):646-674.

10. Lü HM, Zhang MW, Niu LM, Zeng HA, Yan M. Clinical observation of apatinib mesylate for the treatment of multi-drug resistant advanced breast cancer. Zhonghua Yi Xue Za Zhi. 2018;98(16): $1246-1249$.

11. Wu D, Liang L, Nie L, et al. Efficacy, safety and predictive indicators of apatinib after multilines treatment in advanced nonsquamous nonsmall cell lung cancer: Apatinib treatment in nonsquamous NSCLC. Asia Pac J Clin Oncol. Epub 2018 Mar 24.

12. Liang L, Wang L, Zhu P, et al. A pilot study of apatinib as third-line treatment in patients with heavily treated metastatic colorectal cancer. Clin Colorectal Cancer. 2018;17(3):e443-e449.

13. Li L, Xiao S, Zhang L, et al. An open label, single-armed, exploratory study of apatinib (a novel VEGFR-2 tyrosine kinase inhibitor) in patients with relapsed or refractory non-Hodgkin lymphoma. Oncotarget. 2018;9(22):16213-16219.

14. Xie L, Guo W, Wang Y, Yan T, Ji T, Xu J. Apatinib for advanced sarcoma: results from multiple institutions' off-label use in China. BMC Cancer. 2018;18(1):396.

15. Zhu B, Li J, Xie Q, Diao L, Gai L, Yang W. Efficacy and safety of apatinib monotherapy in advanced bone and soft tissue sarcoma: an observational study. Cancer Biol Ther. 2018;19(3):198-204.

16. Miao M, Deng G, Luo S, et al. A phase II study of apatinib in patients with recurrent epithelial ovarian cancer. Gynecol Oncol. 2018;148(2): 286-290.

17. $\mathrm{Wu} \mathrm{H}, \mathrm{Lu} \mathrm{X}, \mathrm{Xu} \mathrm{J}$, et al. A pilot trial assessing apatinib in advanced head and neck squamous cell carcinoma that failed in previous standard chemotherapy or chemoradiotherapy. Int J Radiat Oncol Biol Phys. 2018;102(3):e369.

18. Wu H, Lu X, Xu J, et al. Apatinib plus radiation therapy or not in chemotherapy-refractory recurrent or metastatic oral squamous cell carcinoma: a pilot study. Int J Radiat Oncol Biol Phys. 2018; 102(3):e358.

19. Wang W, Zhang L, Xie Y, Zhen T, Su G, Zang Q. Fatal hemoptysis in patients with advanced esophageal cancer treated with apatinib. Onco Targets Ther. 2018;11:2565-2570.
OncoTargets and Therapy

\section{Publish your work in this journal}

OncoTargets and Therapy is an international, peer-reviewed, open access journal focusing on the pathological basis of all cancers, potential targets for therapy and treatment protocols employed to improve the management of cancer patients. The journal also focuses on the impact of management programs and new therapeutic agents and protocols on

\section{Dovepress}

patient perspectives such as quality of life, adherence and satisfaction. The manuscript management system is completely online and includes a very quick and fair peer-review system, which is all easy to use. Visit http://www.dovepress.com/testimonials.php to read real quotes from published authors. 